\title{
Dumping Syndrome: A Rare Complication Following Nissen Fundoplication
}

\author{
Joana Bernardino Cardoso, David Sousa, Cátia Pereira, João Rua, Ana Teixeira, Jorge Fortuna, Armando Carvalho \\ Centro Hospitalar e Universitário de Coimbra, Coimbra, Portugal
}

Doi: 10.12890/2019_001177 - European Journal of Case Reports in Internal Medicine - ๔ EFIM 2019

\begin{abstract}
Received: 06/06/2019
Accepted: $17 / 06 / 2019$

Published: $10 / 07 / 2019$

How to cite this article: Bernardino Cardoso J, Sousa D, Pereira C, Rua J, Teixeira A, Fortuna J, Carvalho A. Dumping syndrome: a rare complication following Nissen fundoplication. EJCRIM 2019;6: doi:10.12890/2019_001177.
\end{abstract}

Conflicts of Interests: The Authors declare that there are no competing interest

This article is licensed under a Commons Attribution Non-Commercial 4.0 License

\section{ABSTRACT}

Dumping syndrome (DS) is a debilitating entity with gastrointestinal and vasomotor symptoms due to rapid gastric emptying. It is a rare complication of Nissen fundoplication in adults.

We report the case of a patient with oesophageal compressive symptoms (dysphagia, oesophageal food impaction and regurgitation) and frequent episodes of sweating and hypoglycaemia after a Nissen fundoplication to treat gastroesophageal reflux disease. Investigation revealed severe oesophagogastric stenosis and excluded insulinoma and other causes of hypoglycaemia. An oral glucose tolerance test and gastric emptying scintigraphy were performed, supporting the diagnosis of DS.

\section{LEARNING POINTS}

- Dumping syndrome following Nissen fundoplication is a rare condition in adults.

- Diagnosis requires exclusion of other causes of hypoglycaemia.

- First-line treatment is based on dietary modifications, although some patients may require pharmacological therapy.

\section{KEYWORDS}

Dumping syndrome, hypoglycaemia, fundoplication, gastroesophageal reflux

\section{CASE DESCRIPTION}

A 76-year-old male patient with complaints of frequent episodes of profuse sweating and significant weight loss was admitted to our hospital. He first reported the symptoms after a Nissen fundoplication a year previously. After the surgery, he began referring dysphagia, mainly to solids, with oesophageal food impaction and recurrent episodes of regurgitation. He also complained of diarrhoea (faeces without blood or mucus) and a $15 \mathrm{~kg}$ weight loss despite maintained appetite.

Approximately 6 months after the intervention, he started noticing almost daily episodes of profuse sweating, unrelated to food intake or exercise, and never occurring at night. In some of these episodes, hypoglycaemia ( 37 and $50 \mathrm{mg} / \mathrm{dl}$ ) had been identified.

On presentation, his physical examination was unremarkable.

To exclude an underlying insulinoma, a 72-hour fasting test was performed, during which no instance of hypoglycaemia was detected. Insulin and C-peptide assays were both within the normal range. A $50 \mathrm{~g}$ oral glucose tolerance test revealed significantly decreased glycaemia after 1 and 2 hours, although not fulfilling criteria for hypoglycaemia. Autoimmune hypoglycaemia was ruled out by immunological assay.

An oesophageal transit study was ordered to investigate the patient's selective dysphagia, and demonstrated severe gastroesophageal stenosis (Fig. 1). Secondary dumping syndrome (DS) was then considered as the most likely diagnosis. The patient underwent gastric emptying scintigraphy that revealed a greatly decreased gastric emptying half-time of approximately 5 min (normal half-time $>37$ min), confirming the diagnosis (Fig. 2). 


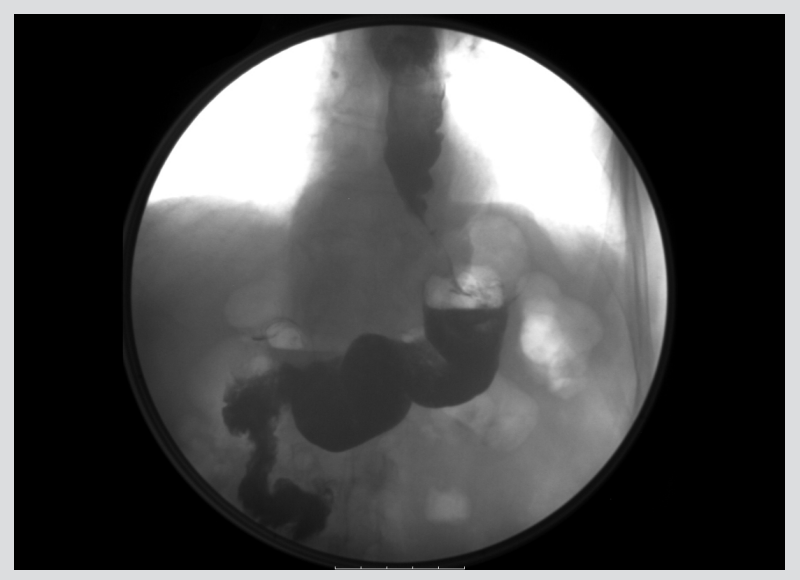

Figure 1. Radiograph of barium oesophageal transit showing severe gastroesophageal stenosis

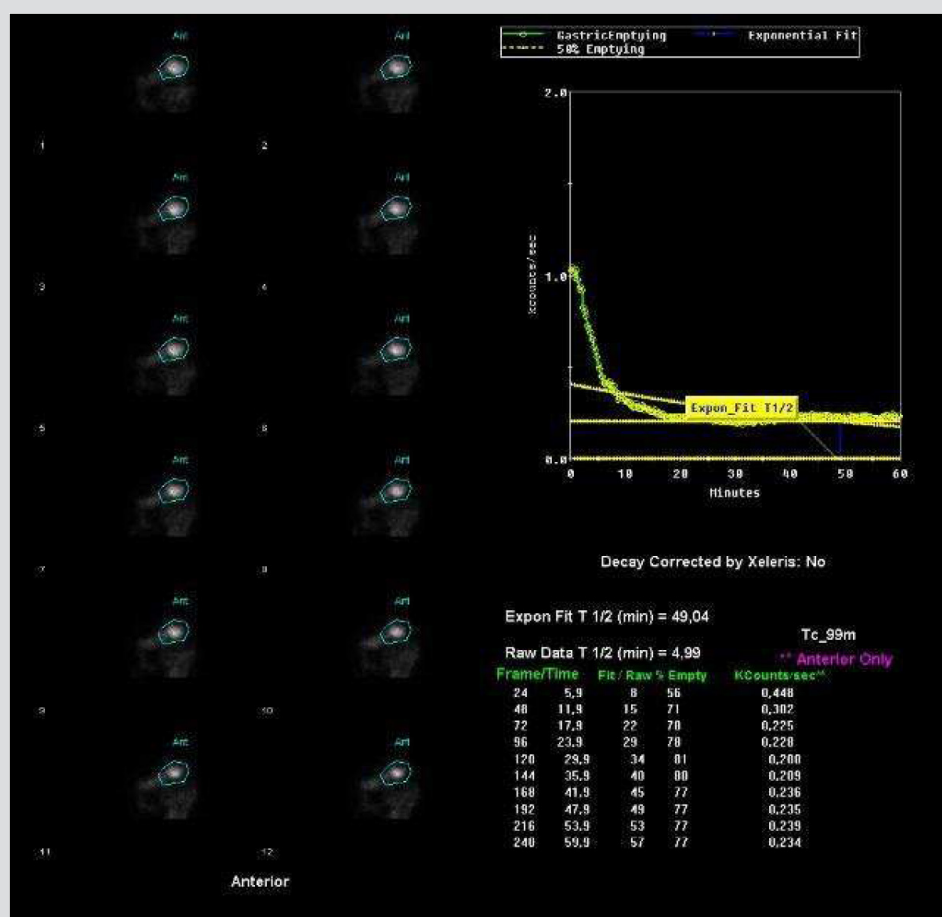

Figure 2. Gastric emptying scintigraphy after radioactive meal ingestion revealed a decreased gastric emptying half-time (normal half-time gastric emptying time is $>37 \mathrm{~min}$ )

Dietary modification, with a low-carbohydrate diet and separation of solid food from liquid ingestion, was instituted. The patient reported improvement in his symptoms with no new episodes of hypoglycaemia or sweating. Endoscopic dilation was performed with clinical improvement of the compressive symptoms and weight recovery.

\section{DISCUSSION}

DS is defined by a constellation of symptoms caused by rapid emptying of nutrients from the stomach into the small intestine, resulting in the release of vasoactive agents and glucose-modulating hormones ${ }^{[1]}$.

The symptoms of DS are classified as either early or late. Early symptoms include systemic (palpitations, tachycardia, fatigue, flushing or pallor, diaphoresis, hypotension, headache and possible syncope) and abdominal manifestations (early satiety, epigastric fullness, abdominal pain, diarrhoea, nausea or cramps), and begin within 30 min following a meal. They occur because of bowel distention, gastrointestinal hormone hypersecretion and autonomic dysregulation. Late symptoms are characterized by perspiration, faintness, decreased concentration and hypoglycaemia, and occur 1-3 hours post-prandially. The proposed pathophysiology indicates that rapid absorption of carbohydrates exaggerates the glucose-mediated insulin response resulting in post-prandial hyperinsulinaemic hypoglycaemia. Patients with late DS often have early DS as well[2]. 
DS is rare after Nissen fundoplication and has predominantly been reported in the paediatric population. An accidental vagal nerve injury during fundoplication surgery has been suggested as a possible pathophysiological determinant, although the exact aetiology is unclear ${ }^{[3]}$. The presence of hypoglycaemia concomitantly with several other symptoms is a strong indicator of DS, although the occurrence of insulinoma needs to be excluded. Gastric emptying scintigraphy and oral glucose tolerance tests are also helpful in diagnosing DS ${ }^{[4]}$.

First-line treatment includes dietary modifications: taking smaller and more frequent meals, ingesting liquids separately from meals, and decreasing monomeric carbohydrate intake. Some patients may require medical therapy with acarbose or octreotide ${ }^{[2]}$. Acarbose is a competitive inhibitor of intestinal brush border $\alpha$-glucosidase that delays the conversion of oligosaccharides to monosaccharides preventing hypoglycaemia. Octreotide is a somatostatin analogue which inhibits several gastrointestinal hormones resulting in delayed gastric emptying, prolonged small intestine transit time and decreased insulin release ${ }^{[5]}$.

This case report is remarkable for describing the difficult challenge of diagnosing the cause of hypoglycaemia, requiring complex studies that revealed DS, a rare complication of Nissen fundoplication in adults.

\section{REFERENCES}

1. Kataria R, Linn S, Malik Z, Abbas AE, Parkman H, Schey R. Post-fundoplication dumping syndrome: a frequent "rare" complication. ACG Case Rep J $2008 ; 5:$ 1.

2. Berg P, McCallum R. Dumping syndrome: a review of the current concepts of pathophysiology, diagnosis, and treatment. Dig Dis Sci 2016;61:11-18.

3. Hejazi RA, Patil H, McCallum RW. Dumping syndrome: establishing criteria for diagnosis and identifying new etiologies. Dig Dis Sci 2010;55:117.

4. Tack J, Arts J, Caenepeel P, De Wulf D, Bisschops R. Pathophysiology, diagnosis and management of postoperative dumping syndrome. Nat Rev Gastroenterol Hepatol 2009;6:583.

5. Hui C, Bauza GJ. Dumping syndrome. StatPearls Publishing; 2019. 